Research Paper

\title{
The Efficacy and Toxicity of Gefitinib in Treating Non-small Cell Lung Cancer: A Meta-analysis of 19 Randomized Clinical Trials
}

\author{
Hongmei $\mathrm{Wo}^{1}$, Jing $\mathrm{He}^{2}$, Yang Zhao ${ }^{2}$, Hao $\mathrm{Yu}^{2}$, Feng Chen ${ }^{2}$ and Honggang $\mathrm{Yi}^{2}{ }^{\varpi}$ \\ 1. Department of Health Economics, School of Health Policy and Management, Nanjing Medical University, Nanjing, 211166, China \\ 2. Department of Biostatistics, School of Public Health, Nanjing Medical University, Nanjing, 211166, China \\ $\triangle$ Corresponding authors: Honggang Yi, M.D., Ph.D., Department of Biostatistics, School of Public Health, Nanjing Medical University, 101 Longmian A.V., \\ Nanjing, Jiangsu, 211166, China; Email: honggangyi@njmu.edu.cn. Feng Chen, PhD, Department of Biostatistics, School of Public Health, Nanjing Medical \\ University, 101 Longmian A.V., Nanjing, Jiangsu, 211166, China; Email: fengchen@njmu.edu.cn \\ (c) Ivyspring International Publisher. This is an open access article distributed under the terms of the Creative Commons Attribution (CC BY-NC) license \\ (https://creativecommons.org/licenses/by-nc/4.0/). See http://ivyspring.com/terms for full terms and conditions.
}

Received: 2017.10.16; Accepted: 2018.02.05; Published: 2018.04.06

\begin{abstract}
Background: This meta-analysis evaluated the efficacy and toxicity of gefitinib with other commonly used drugs in different treatment settings and epidermal growth factor receptor (EGFR) mutation status.

Methods: Nineteen randomize clinical trials (RCTs) of 6,554 patients with NSCLC were pooled in this meta-analysis by random-effects or fixed-effects model, whichever is proper.

Results: In first-line therapy, gefitinib showed higher odds than chemotherapy $(\mathrm{OR}=2.19,95 \% \mathrm{Cl}$ : $1.20-4.01$ ), but less than other targeted therapies (OR $=0.58,95 \% \mathrm{Cl}: 0.38-0.88)$. As non-first-line therapy, the overall survival (OS) and progression-free survival (PFS) were similar between gefitinib and controls $(\mathrm{HR}=1.00,95 \% \mathrm{Cl}: 0.93-1.08 ; \mathrm{HR}=0.91,95 \% \mathrm{Cl}: 0.72-1.15)$, respectively. With the regard to toxicity, the incidences of dry skin, rash and pruritus were higher in gefitinib compared with controls, while gefitinib significantly reduced the incidence of hematologic toxicity.

Conclusion: Gefitinib might be more efficient than chemotherapy, but less efficient than other targeted therapies in ORR, especially in EGFR mutation-positive patients. Gefitinib can decrease the odds of hematologic toxicity compared to controls. Future studies, especially those with EGFR mutation-positive patients, will be needed to confirm our findings.
\end{abstract}

Key words: gefitinib, non-small cell lung cancer, meta-analysis, efficacy, toxicity.

\section{Introduction}

Lung cancer is one of the most common malignant tumors which is the major causes of cancer death worldwide. With a serious impact on one's mental and physical health as well as quality of life, lung cancer has become a global health problem [1]. Non-small cell lung cancer (NSCLC) accounts for approximately $80 \%$ of lung cancer cases, including adenocarcinoma, squamous carcinoma and so on [2]. Lung cancer is diagnosed at an advanced stage in $30-40 \%$ of patients, which causes losing the most effective timing for surgery, and therefore high mortality [3]. Unfortunately, conventional chemoradiotherapy has poor sensitivity and significant adverse effects. Platinum-based doublet chemotherapy is the standard first-line treatment for advanced NSCLC, while its effective rate remains only $30-40 \%$ [4]. In addition, patients who failed first-line therapy have few effective treatment options for the second-line therapy [5]. Thus, the development of more effective and safer therapy is badly needed.

Recent advances in genetic discoveries have proved that epidermal growth factor receptor (EGFR) -dependent pathway is activated in more than half of the patients with NSCLC and it plays an important 
role in the development and progression of epithelial cells. Also, molecular targeted drugs appear constantly, providing a new idea for tumor treatment [6]. EGFR-tyrosine kinase inhibitor (EGFR-TKI) is a small-molecule drug that targets the active adenosine triphosphate binding (ATP) site of EGFR kinase. Gefitinib was one of the first-generation EGFR-TKIs in clinical practice, and has been listed in National Comprehensive Cancer Network (NCCN) guidelines for the treatment of NSCLC [7]. Relevant clinical trials have been designed to determine its efficacy and toxicity; however, different research designs, limited case numbers, and failure to analyze the effect of EGFR mutation are major concerns. In addition, quality of the research has not been evaluated systematically and thoroughly.

Recently, systematic reviews have been investigated to explore the efficacy and/or toxicity of gefitinib. One meta-analysis [8] synthesized 56 randomized controlled trials (RCTs) to elucidate optimal first-line therapies for advanced NSCLC, but only focused on the first-line with maintenance therapy, instead of the monotherapy with gefitinib. Two other meta-analyses also evaluated the efficiency of EGFR inhibitors $[9,10]$. However, both of them included limited numbers of RCTs. Four RCTs addressed the effect of EGFR-activating mutations in one meta-analysis [10], and gefitinib was only assessed among two RCTs in the other [9]. Moreover, one study didn't compare the toxicities of gefitinib with other agents [10], and the other one only focused on first-line therapy [9]. As for the toxicity, one meta-analysis evaluated the incidence of pulmonary toxicity based on 23 RCTs [11]. However, other toxicities, such as hematologic toxicity and abnormal hepatic function, were not evaluated and remained unclear.

To our best knowledge, gefitinib for the EGFR-mutated and non-mutated patients have not been systematically evaluated as both the first-line and non-first-line therapy of NSCLC, compared with placebo, chemotherapy and other targeted therapies. Therefore, in order to obtain the best evidence, a meta-analysis of RCTs comparing gefitinib with other agent-based regimens of NSCLC on their efficacies and toxicities.

\section{Methods}

\section{Search strategy}

The aim of this meta-analysis was to review all published and reported RCTs comparing the gefitinib with other agent-based regimens. All relevant articles were retrieved by internet searching through the PubMed database, the Cochrane Central Register of
Controlled Trials (CENTRAL), the China National Knowledge Infrastructure (CNKI), and the VIP citation database, with the various combinations of the following terms: gefitinib, non-small cell lung cancer, NSCLC, and randomized controlled trial. The published language was limited to English and Chinese, and the years were limited from 1999 to July 2017. In addition, we searched trial registries, conference proceedings, reference lists of original articles, and contacted original trialists for possible unpublished trials.

\section{Inclusion and exclusion criteria}

The relevant RCTs were eligible for inclusion in the meta-analysis if they met all following criteria: (1) They dealt with patients histologically or cytologically confirmed NSCLC and $\geq 18$ years old; (2) Intervention: gefitinib as monotherapy for the treatment of NSCLC; (3) Control: placebo, chemotherapy without gefitinib or other targeted therapy; (4) Endpoints: In first-line therapy, the endpoints included objective response rate (ORR) and with their 95\% confidence intervals (CIs). In non-first-line therapy (including second-line, third-line or maintenance therapy), the endpoint were overall survival (OS) and progression-free survival (PFS); (5) All included trials must be RCTs. Studies failing to meet the inclusion criteria, studies with missing adequate statistical analysis information, sub-analysis, post-hoc analysis, reviews, animal experiments and phase I trials will be excluded.

\section{Data extraction and quality assessment}

All potentially relevant articles were reviewed by two independent reviewers. The references were screened by titles and further selected by reading the abstracts. For those articles that were not excluded based on title and abstract, reviewers retrieved full text, made judgments and decided final conclusion for them. Disagreements were resolved by discussion or consulting a third reviewer after referring to the original articles. Multiple references of the same study were considered as one publication, and only the most recent or complete study was examined. Articles were also obtained from cross-checking references of publications.

Data was also independently extracted from each articles by two reviewers, who were blinded to each other, using standardized data abstraction forms, including: (1) basic information of the included studies (such as the first author, year of publication, and location in which the study was performed); (2) trial characteristics (such as number of patients, study design, details of therapeutic regimens, and follow-up); (3) patients' characteristics (such as median age, sex, smoking status, disease stage, 
pathological subtype and EGFR mutation), (4) Information related to the quality of trials; (5) efficiency and toxicity outcome measures, including ORR, median PFS and OS, hazard ratios (HRs) for PFS and OS, and adverse events. For ORRs, the reported number of objective response (complete response $(\mathrm{CR})+$ partial response (PR)) and no response in each arm was collected. All data were checked for internal consistency, and any disagreements were resolved by discussion among the investigators.

The quality of the study was independently accessed by two reviewers, and any disagreements were resolved by consensus. All included studies were evaluated using the Cochrane Collaboration Tool considering six criteria [12].

\section{Outcome Measures}

In first-line therapy, the primary endpoint was ORR, which was defined as the proportion of CR plus PR among evaluable patients. In non-first-line therapy, the endpoints were OS and PFS. PFS is defined as the duration of time from random assignment to documented disease progression or death, whichever occurs first. OS is defined as the time from random assignment to death, irrespective of the cause of death.

\section{Statistical Analysis}

Except adverse events, most analyses were performed according to the intention-to-treat (ITT) principle. For OS and PFS, the pooled HRs with 95\% CIs were calculated based on the log HRs using as general variance-based method [13]. A HR value greater than 1 represented higher death and tumor progress risk in gefitinib than in controls. For ORR and adverse events, the pooled ORs were calculated. An OR value greater than 1 indicates a higher tumor response rate or more toxicity in gefitinib than in controls.

We first assessed the variability across studies attributable to heterogeneity beyond chance using the Cochran's $Q$-test and $I^{2}$ statistic. The clinical trials were considered heterogeneous when the $P$ value from $Q$-test was $\leq 0.10$, or $I^{2}$ was $>50 \%$. When there was no statistically significant heterogeneity, a pooled effect was calculated with a fixed-effects model; otherwise, a random-effects model was applied to accommodate the heterogeneity [14]. Sensitivity analysis was also conducted to determine if the results were influenced by excluding the study with the largest sample size, EGFR mutation status, or the severity of AE.

Finally, the potential publication bias among trials was evaluated visually by funnel plot, and statistically by the Begg's test and the Egger's test. If the publication bias existed, Duval and Tweedie's "trim and fill" method (TFM) was used to estimate the associations of interest accordingly [15-17].

All statistical analyses were performed with the Cochrane Collaboration's Review Manager V.5.3.5 (the Cochrane Collaboration, Oxford, UK) and STATA 13.0 (STATA Corporation, College Station, TX). All CIs had two-sided probability coverage of $95 \%$. All statistical tests were two-sided, and a $P$ value $\leq 0.05$ was considered as significant, if not otherwise specified.

The study was performed according to the Preferred Reporting Items for Systematic Reviews and Meta-Analyses (PRISMA) statement [15-17].

\section{Results}

\section{Characteristics of included studies}

The comprehensive search of PubMed, CENTRAL, CNKI, and VIP initially yielded 432 articles, and nineteen studies with 6,554 participants had been published met the pre-determined inclusion criteria and were included (Figure 1).

Among the all nineteen RCTs, eight of the clinical trials were first-line therapy [19-26], the rests were non-first-line therapy [26-36] (Table 1). Overall, 6,554 patients were randomized to receive gefitinib (3,280 patients) or other agent-based regimens $(3,274$ patients). A total of 28 patients were excluded after randomization. The patient level analyses showed patient median age varied from 55 to 76 years, percent of male varied from $10.7 \%$ to $78 \%$, percent of adenocarcinoma varied from 35.1 to $100 \%$, and 58.1 $100 \%$ of patients having cancer stage higher than III across trials (Table 2).

\section{Quality assessment}

The summary of study quality showed that all nineteen RCTs applied randomization technique, but nine of them didn't provide details [19, 20, 22, 24, 25, $27,30,31,37]$. Ten RCTs applied sufficient allocation concealment, and double-blind method was adopted only in five RCTs [20, 30, 32, 33, 35]. One RCT resulted in early termination because gefitinib can statistically improve PFS as compared with standard chemotherapy in planned interim analysis (Figure S1) [22].

\section{Efficacy analysis}

Objective response rate (ORR). Eight RCTs with 2,637 patients (gefitinib, $n=1,323$; controls, $n=1,314$ ) were included in first-line therapy. The heterogeneity among these trials was significant $\left(P<0.001, I^{2}=86 \%\right)$ and a random-effects model was used. Gefitinib showed higher ORR than chemotherapy $(\mathrm{OR}=2.19$, 95\% CI: 1.20-4.01), lower ORR than other targeted 


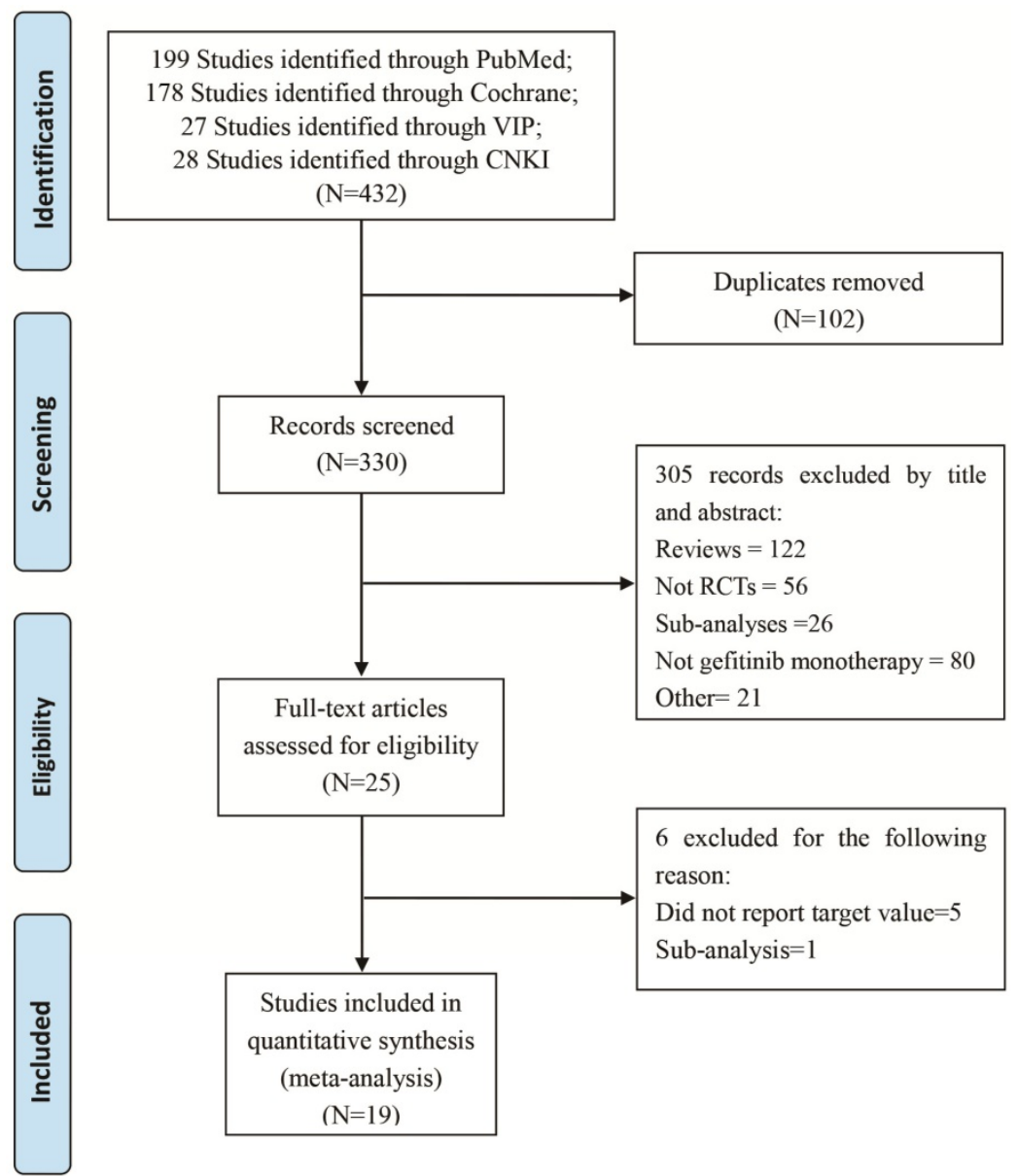

Figure 1. PRISMA flow diagram of eligible studies for the meta-analysis of gefitinib compared to controls for NSCLC. Abbreviations: NSCLC: non-small cell lung cancer.

therapies (OR $=0.57,95 \% \mathrm{CI}: 0.38-0.86, P=0.010)$, and there was no significant improvement of ORR comparing with placebo $(\mathrm{OR}=6.38,95 \% \mathrm{CI}$ : 0.75-54.02), respectively (Figure 2).

Overall survival (OS). Eleven RCTs with 3,585 patients (gefitinib, $n=1,801$; other agents, $n=1,784$ ) were available for OS as non-first-line therapy. The $I^{2}$ test for heterogeneity was $7 \%(P=0.380)$, which didn't show any major qualitative evidence for heterogeneity among studies. The results of a fixed-effects model showed no significant difference in OS comparing gefitinib with placebo $(\mathrm{HR}=0.83$, 95\% CI: 0.66-1.03), chemotherapy ( $\mathrm{HR}=1.03,95 \% \mathrm{CI}$ : 0.94-1.13), or other targeted therapies ( $\mathrm{HR}=1.00,95 \%$ CI: 0.86-1.17), respectively (Figure 3).

Progression-free survival (PFS). The test of heterogeneity showed a significant difference $(P<$ $\left.0.001, I^{2}=89 \%\right)$, so we used a random-effects model and the results showed that gefitinib increased PFS $(\mathrm{HR}=0.50,95 \% \mathrm{CI}: 0.35-0.72)$ compared with placebo, and showed a similar PFS compared with chemotherapy $(\mathrm{HR}=0.94, \quad 95 \%$ CI: 0.73-1.22). However, gefitinib showed a decreased PFS compared with other targeted therapies $(\mathrm{HR}=1.26$, 95\% CI: 1.08-1.46) (Figure 4).

\section{EGFR Mutation subgroup analysis}

Ten trails and 1,408 patients with EGFR mutation testing were available in subgroup analysis, which were grouped into EGFR mutation-positive subgroup with 997 patients and EGFR mutation-negative subgroup with 411 patients. For the first-line therapy in EGFR mutation-positive subgroup, patients using gefitinib had greater odds of having higher ORR compared with chemotherapy $(\mathrm{OR}=4.23,95 \%$ CI: 2.17-8.28), but had less odds compared with other targeted therapies $(\mathrm{OR}=0.58$, 95\% CI: 0.38-0.88). There is no significant difference between gefitinib with controls in EGFR mutation-negative subgroup $(\mathrm{OR}=0.33,95 \% \mathrm{CI}$ : 0.10-1.02). For the non-first-line therapy, neither EGFR mutation-positive group nor mutation-negative group showed significant results in PFS or OS between gefitinib and controls (Table S1 and Table S2). 
Table 1. Selected characteristics of 19 studies included in the meta-analysis of gefitinib compared to controls for NSCLC

\begin{tabular}{|c|c|c|c|c|c|c|c|c|c|c|c|c|c|}
\hline \multirow[t]{2}{*}{ Trial } & \multirow[t]{2}{*}{ Region } & \multirow[t]{2}{*}{ Design } & \multirow[t]{2}{*}{ Treatment Arms } & \multicolumn{4}{|c|}{ Number of Patients } & \multirow{2}{*}{$\begin{array}{l}\text { ITT } \\
\text { Analysis }\end{array}$} & \multirow{2}{*}{$\begin{array}{l}\text { Median } \\
\text { Age } \\
\text { (Year) }\end{array}$} & \multirow{2}{*}{$\begin{array}{l}\text { Sex } \\
\text { (Male, } \\
\%)\end{array}$} & \multirow{2}{*}{$\begin{array}{l}\text { Smoking } \\
\text { History (\%) }\end{array}$} & \multirow{2}{*}{$\begin{array}{l}\text { Stage } \\
\text { III/IV } \\
(\%)\end{array}$} & \multirow{2}{*}{$\begin{array}{l}\text { Adenocar- } \\
\text { cinoma (\%) }\end{array}$} \\
\hline & & & & $\begin{array}{l}\text { Rando } \\
\text { mized }\end{array}$ & Excluded & $\begin{array}{l}\text { Loss of } \\
\text { follow- } \\
\text { up }\end{array}$ & $\begin{array}{l}\text { ITT } \\
\text { Population }\end{array}$ & & & & & & \\
\hline \multicolumn{14}{|c|}{ First-line Therapy } \\
\hline \multirow[t]{2}{*}{$\begin{array}{l}\text { Crinò et al, } \\
2008\end{array}$} & $\begin{array}{l}\text { Australia, Brazil, } \\
\text { Czech Republic, }\end{array}$ & $\begin{array}{l}\text { RCT, open } \\
\text { label }\end{array}$ & $\begin{array}{l}\text { Gefitinib 250mg/d, } \\
\text { po }\end{array}$ & 97 & 0 & 0 & 97 & Yes & 74 & 77.3 & 82.5 & 100 & 35.1 \\
\hline & $\begin{array}{l}\text { France, Germany, } \\
\text { Italy, Republic of } \\
\text { Korea, South } \\
\text { Africa, Taiwan } \\
\text { and United } \\
\text { Kingdom }\end{array}$ & & $\begin{array}{l}\text { Vinorelbine } 30 \\
\mathrm{mg} / \mathrm{m}^{2}\end{array}$ & 99 & 0 & 0 & 99 & & 74 & 73.7 & 88.9 & 100 & 45.5 \\
\hline \multirow[t]{2}{*}{$\begin{array}{l}\text { Goss et al, } \\
2009\end{array}$} & $\begin{array}{l}\text { Australia, Canada, } \\
\text { Czech Republic, }\end{array}$ & $\begin{array}{l}\text { RCT, } \\
\text { double-blind }\end{array}$ & $\begin{array}{l}\text { Gefitinib } 250 \mathrm{mg} / \mathrm{d} \text {, } \\
\text { po }\end{array}$ & 100 & 0 & 0 & 100 & Yes & 74 & 61 & 90 & NR & 45 \\
\hline & $\begin{array}{l}\text { Netherlands and } \\
\text { United Kingdom }\end{array}$ & & Placebo & 101 & 0 & 0 & 101 & & 76 & 60.4 & 91.1 & NR & 45.5 \\
\hline \multirow[t]{2}{*}{$\begin{array}{l}\text { Mok et al, } \\
2009\end{array}$} & Asia & $\begin{array}{l}\text { RCT, open } \\
\text { label }\end{array}$ & $\begin{array}{l}\text { Gefitinib } 250 \mathrm{mg} / \mathrm{d} \text {, } \\
\text { po }\end{array}$ & 609 & 0 & 5 & 609 & Yes & 57 & 20.5 & 6.3 & 100 & 95.4 \\
\hline & & & $\begin{array}{l}\text { Paclitaxel } 200 \mathrm{mg} / \mathrm{m}^{2} \\
\text { plus Carboplatin, i.v. }\end{array}$ & 608 & 0 & 2 & 608 & & 57 & 20.9 & 6.4 & 99.8 & 97.2 \\
\hline \multirow[t]{2}{*}{$\begin{array}{l}\text { Maemondo } \\
\text { et al, } 2010\end{array}$} & Japan & $\mathrm{RCT}$ & $\begin{array}{l}\text { Gefitinib } 250 \mathrm{mg} / \mathrm{d} \text {, } \\
\text { po }\end{array}$ & 115 & 1 & 0 & 114 & Yes & 63.9 & 36.8 & 34.2 & 90.4 & 90.4 \\
\hline & & & $\begin{array}{l}\text { Paclitaxel } 200 \mathrm{mg} / \mathrm{m}^{2} \\
\text { plus Carboplatin, i.v. }\end{array}$ & 115 & 1 & 0 & 114 & & 62.6 & 36 & 42.1 & 92.1 & 96.5 \\
\hline \multirow[t]{2}{*}{$\begin{array}{l}\text { Mitsudomi } \\
\text { et al, } 2010\end{array}$} & Japan & $\begin{array}{l}\text { RCT, } \\
\text { open-label }\end{array}$ & $\begin{array}{l}\text { Gefitinib } 250 \mathrm{mg} / \mathrm{d} \text {, } \\
\text { po }\end{array}$ & 88 & 2 & 0 & 86 & Yes & 64 & 31.4 & 29.1 & 59.3 & 96.5 \\
\hline & & & $\begin{array}{l}\text { Cisplatin } 80 \mathrm{mg} / \mathrm{m}^{2}+ \\
\text { Docetaxel } 60 \mathrm{mg} / \mathrm{m}^{2} \text {, } \\
\text { i.v. }\end{array}$ & 89 & 3 & 0 & 86 & & 64 & 30.2 & 33.7 & 58.1 & 97.7 \\
\hline \multirow[t]{2}{*}{$\begin{array}{l}\text { Han et al, } \\
2012\end{array}$} & Korean & $\begin{array}{l}\mathrm{RCT} \text {, } \\
\text { open-label }\end{array}$ & $\begin{array}{l}\text { Gefitinib } 250 \mathrm{mg} / \mathrm{d} \text {, } \\
\text { po }\end{array}$ & 159 & 0 & 0 & 159 & Yes & 57 & 12 & 0 & 100 & 100 \\
\hline & & & $\begin{array}{l}\text { Gemcitabine } 1,250 \\
\mathrm{mg} / \mathrm{m}^{2} \text { plus Cisplatin } \\
80 \mathrm{mg} / \mathrm{m}^{2} \text {, i.v. }\end{array}$ & 154 & 4 & 0 & 150 & & 56.5 & 10.7 & 0 & 100 & 100 \\
\hline \multirow[t]{2}{*}{$\begin{array}{l}\text { Xie et al, } \\
2015\end{array}$} & China & $\mathrm{RCT}$ & $\begin{array}{l}\text { Gefitinib } 250 \mathrm{mg} / \mathrm{d} \text {, } \\
\text { po }\end{array}$ & 27 & 0 & 0 & 27 & Yes & 62.5 & 37 & NR & 100 & NR \\
\hline & & & $\begin{array}{l}\text { Erlotinib } 150 \mathrm{mg} / \mathrm{d} \text {, } \\
\text { po. }\end{array}$ & 23 & 0 & 0 & 23 & & 62.5 & 43.5 & NR & 100 & NR \\
\hline \multirow[t]{2}{*}{$\begin{array}{l}\text { Park et al, } \\
2016\end{array}$} & $\begin{array}{l}\text { Australia, Canada, } \\
\text { China, France, }\end{array}$ & $\begin{array}{l}\text { RCT, } \\
\text { open-label }\end{array}$ & $\begin{array}{l}\text { Gefitinib } 250 \mathrm{mg} / \mathrm{d} \text {, } \\
\text { po }\end{array}$ & 159 & 0 & 0 & 159 & Yes & 63 & 33.3 & 33.3 & 100 & 99.4 \\
\hline & $\begin{array}{l}\text { Germany, Ireland, } \\
\text { Norway, Republic } \\
\text { of Korea, } \\
\text { Singapore, Spain, } \\
\text { Sweden, Taiwan, } \\
\text { and United } \\
\text { Kingdom }\end{array}$ & & Afatinib $40 \mathrm{mg} / \mathrm{d}$, po & 160 & 0 & 0 & 160 & & 63 & 43.1 & 33.8 & 100 & 99.4 \\
\hline \multicolumn{14}{|c|}{ Non-first-line Therapy } \\
\hline \multirow[t]{2}{*}{$\begin{array}{l}\text { Cufer et al, } \\
2006\end{array}$} & $\begin{array}{l}\text { Europe, South } \\
\text { American and }\end{array}$ & $\begin{array}{l}\mathrm{RCT} \text {, open } \\
\text { label }\end{array}$ & $\begin{array}{l}\text { Gefitinib } 250 \mathrm{mg} / \mathrm{d} \text {, } \\
\text { po }\end{array}$ & 68 & 0 & NR & 68 & Yes & 63 & 69 & 67.6 & 100 & NR \\
\hline & $\begin{array}{l}\text { Middle-Eastern } \\
\text { countries }\end{array}$ & & $\begin{array}{l}\text { Docetaxel } 75 \mathrm{mg} / \mathrm{m}^{2} \text {, } \\
\text { i.v. }\end{array}$ & 73 & 2 & NR & 73 & & 59.5 & 70 & 67.1 & 100 & NR \\
\hline \multirow[t]{2}{*}{$\begin{array}{l}\text { Maruyama } \\
\text { et al, } 2008\end{array}$} & Japan & $\begin{array}{l}\text { RCT, open } \\
\text { label }\end{array}$ & $\begin{array}{l}\text { Gefitinib } 250 \mathrm{mg} / \mathrm{d} \text {, } \\
\text { po }\end{array}$ & 245 & 1 & 0 & 245 & Yes & NR & 61.6 & 71 & 84.1 & 78.4 \\
\hline & & & $\begin{array}{l}\text { Docetaxel } 60 \mathrm{mg} / \mathrm{m}^{2} \text {, } \\
\text { i.v. }\end{array}$ & 244 & & 0 & 244 & & NR & 61.9 & 64.3 & 82 & 77 \\
\hline \multirow[t]{2}{*}{$\begin{array}{l}\text { Kim et al, } \\
2008\end{array}$} & $\begin{array}{l}\text { Europe, Asia, } \\
\text { America }\end{array}$ & $\begin{array}{l}\text { RCT, open } \\
\text { label }\end{array}$ & $\begin{array}{l}\text { Gefitinib } 250 \mathrm{mg} / \mathrm{d} \text {, } \\
\text { po }\end{array}$ & 733 & 0 & NR & 733 & No & 61 & 63.6 & 79.8 & 90 & 53.9 \\
\hline & & & $\begin{array}{l}\text { Docetaxel } 75 \mathrm{mg} / \mathrm{m}^{2} \text {, } \\
\text { i.v. }\end{array}$ & 733 & 0 & NR & 733 & & 60 & 66.6 & 79.6 & 90.4 & 54.8 \\
\hline \multirow[t]{2}{*}{$\begin{array}{l}\text { Natale et al, } \\
2009\end{array}$} & $\begin{array}{l}\text { Argentina, } \\
\text { Belgium, }\end{array}$ & $\begin{array}{l}\text { RCT, } \\
\text { double-blind }\end{array}$ & $\begin{array}{l}\text { Gefitinib } 250 \mathrm{mg} / \mathrm{d} \text {, } \\
\text { po }\end{array}$ & 85 & 0 & 0 & 85 & Yes & 61 & 61.2 & 90.6 & 100 & 62.4 \\
\hline & $\begin{array}{l}\text { Germany, South } \\
\text { Africa, United } \\
\text { Kingdom and the } \\
\text { United State }\end{array}$ & & $\begin{array}{l}\text { Vandetanib } 300 \\
\mathrm{mg} / \mathrm{d} \text {, po }\end{array}$ & 83 & 0 & 0 & 83 & & 63 & 57.8 & 85.5 & 100 & 54.2 \\
\hline $\begin{array}{l}\text { Lee } e t \text { al, } \\
2010\end{array}$ & Korea & $\begin{array}{l}\text { RCT, open } \\
\text { label }\end{array}$ & $\begin{array}{l}\text { Gefitinib } 250 \mathrm{mg} / \mathrm{d} \text {, } \\
\text { po }\end{array}$ & 82 & 0 & 0 & 82 & Yes & 57 & 67.1 & 63.4 & 100 & 65.9 \\
\hline & & & $\begin{array}{l}\text { Docetaxel } 75 \mathrm{mg} / \mathrm{m}^{2} \text {, } \\
\text { i.v. }\end{array}$ & 79 & 0 & 0 & 79 & & 58 & 57 & 54.4 & 100 & 69.6 \\
\hline $\begin{array}{l}\text { Gaafar et } \\
\text { al., } 2011\end{array}$ & $\begin{array}{l}\text { Belgium, Cyprus, } \\
\text { Egypt, Italy and }\end{array}$ & $\begin{array}{l}\text { RCT, } \\
\text { double-blind }\end{array}$ & $\begin{array}{l}\text { Gefitinib } 250 \mathrm{mg} / \mathrm{d} \text {, } \\
\text { po }\end{array}$ & 86 & 0 & 0 & 86 & Yes & 61 & 78 & 78 & 100 & 57 \\
\hline & Netherlands & & Placebo, po & 87 & 0 & 1 & 87 & & 62 & 76 & 74 & 100 & 46 \\
\hline $\begin{array}{l}\text { Zhang et al, } \\
2012\end{array}$ & China & $\begin{array}{l}\text { RCT, } \\
\text { double-blind }\end{array}$ & $\begin{array}{l}\text { Gefitinib } 250 \mathrm{mg} / \mathrm{d} \text {, } \\
\text { po }\end{array}$ & 148 & 0 & 1 & 148 & Yes & 55 & 56 & 47 & 100 & 71 \\
\hline & & & Placebo, po & 148 & 0 & 1 & 148 & & 55 & 62 & 45 & 100 & 70 \\
\hline
\end{tabular}




\begin{tabular}{|c|c|c|c|c|c|c|c|c|c|c|c|c|c|}
\hline \multirow[t]{2}{*}{ Trial } & \multirow[t]{2}{*}{ Region } & \multirow[t]{2}{*}{ Design } & \multirow[t]{2}{*}{ Treatment Arms } & \multicolumn{4}{|c|}{ Number of Patients } & \multirow{2}{*}{$\begin{array}{l}\text { ITT } \\
\text { Analysis }\end{array}$} & \multirow{2}{*}{$\begin{array}{l}\text { Median } \\
\text { Age } \\
\text { (Year) }\end{array}$} & \multirow{2}{*}{$\begin{array}{l}\text { Sex } \\
\text { (Male, } \\
\%)\end{array}$} & \multirow{2}{*}{$\begin{array}{l}\text { Smoking } \\
\text { History (\%) }\end{array}$} & \multirow{2}{*}{$\begin{array}{l}\text { Stage } \\
\text { III/IV } \\
(\%)\end{array}$} & \multirow{2}{*}{$\begin{array}{l}\text { Adenocar- } \\
\text { cinoma (\%) }\end{array}$} \\
\hline & & & & $\begin{array}{l}\text { Rando } \\
\text { mized }\end{array}$ & Excluded & $\begin{array}{l}\text { Loss of } \\
\text { follow- } \\
\text { up }\end{array}$ & $\begin{array}{l}\text { ITT } \\
\text { Population }\end{array}$ & & & & & & \\
\hline \multirow[t]{2}{*}{$\begin{array}{l}\text { Sun et al, } \\
2012\end{array}$} & Korea & $\begin{array}{l}\text { RCT, open } \\
\text { label }\end{array}$ & $\begin{array}{l}\text { Gefitinib } 250 \mathrm{mg} / \mathrm{d} \text {, } \\
\text { po }\end{array}$ & 71 & 3 & 0 & 68 & No & 58 & 14.7 & 0 & 100 & 100 \\
\hline & & & $\begin{array}{l}\text { Pemetrexed } 500 \\
\mathrm{mg} / \mathrm{m}^{2}, \text { i.v. }\end{array}$ & 70 & 3 & 1 & 67 & & 64 & 14.9 & 0 & 100 & 100 \\
\hline \multirow[t]{2}{*}{$\begin{array}{l}\text { Shi et al, } \\
2013\end{array}$} & China & $\begin{array}{l}\text { RCT, } \\
\text { double-blind }\end{array}$ & $\begin{array}{l}\text { Gefitinib } 250 \mathrm{mg} / \mathrm{d} \text {, } \\
\text { po }\end{array}$ & 199 & 3 & 0 & 196 & Yes & 57 & 56.6 & 48 & 100 & 76.5 \\
\hline & & & $\begin{array}{l}\text { Icotinib, } 125 \mathrm{mg} \text {, tid, } \\
\text { po }\end{array}$ & 200 & 1 & 0 & 199 & & 57 & 58.8 & 49.2 & 100 & 74.9 \\
\hline \multirow[t]{2}{*}{$\begin{array}{l}\text { Zhou et al, } \\
2014\end{array}$} & China & $\begin{array}{l}\text { RCT, } \\
\text { open-label }\end{array}$ & $\begin{array}{l}\text { Gefitinib 250mg/d, } \\
\text { po }\end{array}$ & 81 & 0 & 1 & 81 & Yes & 57.5 & 66.7 & 59.3 & 100 & 97.5 \\
\hline & & & $\begin{array}{l}\text { Pemetrexed } 500 \\
\mathrm{mg} / \mathrm{m}^{2} \text {, i.v. }\end{array}$ & 80 & 4 & & 76 & & 55.9 & 61.8 & 42.1 & 100 & 94.7 \\
\hline \multirow[t]{2}{*}{$\begin{array}{l}\text { Yang et al, } \\
2017\end{array}$} & China & $\mathrm{RCT}$ & $\begin{array}{l}\text { Gefitinib } 250 \mathrm{mg} / \mathrm{d} \text {, } \\
\text { po }\end{array}$ & 128 & 0 & 0 & Yes & 128 & 58.5 & 46.1 & 27.3 & 100 & 96.1 \\
\hline & & & $\begin{array}{l}\text { Erlotinib } 150 \mathrm{mg} / \mathrm{d} \text {, } \\
\text { po }\end{array}$ & 128 & 0 & 0 & & 128 & 58.5 & 46.9 & 18 & 100 & 96.1 \\
\hline
\end{tabular}

Abbreviations: NSCLC: non-small cell lung cancer; RCT: randomized clinical trial; ITT: intention-to-treat; NR: not reported; d: day; po: per oral; i.v.: intravenous injection; tid: three times a day.

\section{Toxicity analysis}

Reported toxicities were analyzed in all nineteen trials. Dry skin (OR $=10.82,95 \% \mathrm{CI}$ : 4.91-23.87), grade $3 / 4$ abnormal hepatic function $(\mathrm{OR}=8.98,95 \% \mathrm{CI}$ : 3.55-22.73), grade $3 / 4$ rash $(\mathrm{OR}=4.27,95 \% \mathrm{CI}$ : 2.75-6.64), pruritus ( $\mathrm{OR}=4.00,95 \% \mathrm{CI}: 2.10-7.62)$, grade $3 / 4$ diarrhea $(\mathrm{OR}=1.49$, 95\% CI: $1.02-2.16)$ were significantly prominent in gefitinib groups than in other agent-based regimens (Table S3).

Interestingly, hematologic toxicity were significant decreased in patients using gefitinib, including leukopenia $(\mathrm{OR}=0.19,95 \% \mathrm{CI}$ : 0.05-0.71), neutropenia $(\mathrm{OR}=0.03,95 \% \mathrm{CI}: 0.02-0.04)$, and anemia $(\mathrm{OR}=0.25,95 \%$ CI: 0.13-0.46). For nonhematologic toxicity, alopecia, neurotoxicity, anorexia and so on, were also significantly decreased in gefitinib groups. Compared to other agent-based regimens, gefitinib did not increase the frequency of other adverse events (Table S3).

\section{Publication bias}

The funnel plot, the Begg's test and the Egger's test suggested that there was no significant publication bias for ORR (Begg's test, $P=1.000$; Egger's test, $P=0.753$ ), OS (Begg's test, $P=0.876$; Egger's test, $P=0.750$ ), and PFS (Begg's test, $P=1.000$; Egger's test, $P=0.580$ ). Trim and fill method showed that one study was missed in the ORR, as represented by hollow circles in Figure S2. However, the conclusion did not change significantly before and after the analysis. As regards OS, the symmetric plot was further observed. Results of TFM showed that no significant alteration to the data when 2 missing studies were added. For PFS, the TFM showed one study was imputed, and suggested that the conclusion did not change significantly before and after the analysis (Figure S2).

\section{Sensitivity analysis}

Sensitivity analyses were performed for ORR, PFS by excluding the study with the largest sample size in the consideration of high heterogeneity, and the results were not materially changed. Subgroup analysis showed that the results were not substantially modified by EGFR mutation status and the severity of AE.

\section{Discussion}

Gefitinib is the first molecular targeted agent for NSCLC, by targeting EGFR inhibiting tyrosine kinase, with adenosine triphosphate (ATP) in receptor tyrosine kinase (RTK) of the catalytic center of Mg-ATP binding site in competitive combination, can effectively inhibit the activity of tyrosine kinase, block the intracellular tyrosine kinase phosphorylation, suspend the EGFR signal conduction, and thus block growth of tumor cell [38].

Platinum-based chemotherapy is a commonly used method of treatment for advanced NSCLC. However, chemotherapy can cause great damage to both body and psychology of patients, eventually leading to significant decrease of survival rate and survival time [39].

Gefitinib has its advantages when compared to chemotherapy, and its application in the treatment of NSCLC need more reliable evidence to support. This study systematically evaluates the efficiency and toxicity of RCTs for gefitinib on NSCLC. Our findings demonstrate that gefitinib could be more efficient than chemotherapy, but less efficient than other targeted therapies in ORR, especially in EGFR mutation-positive patients. However, there is no evidence that gefitinib could improve PFS or OS compared with chemotherapy and other targeted therapies. Furthermore, gefitinib can decrease the odds of hematologic toxicity compared to controls. 
Table 2. Results of outcomes in the meta-analysis of gefitinib compared to controls for NSCLC

\begin{tabular}{|c|c|c|c|c|c|c|c|c|c|c|c|c|c|c|}
\hline \multirow[t]{2}{*}{ Trial } & \multirow{2}{*}{$\begin{array}{l}\text { Therapy } \\
\text { Regimen }\end{array}$} & \multirow{2}{*}{$\begin{array}{l}\text { ITT } \\
\text { Population }\end{array}$} & \multicolumn{5}{|c|}{ ORR } & \multicolumn{3}{|l|}{ PFS } & \multicolumn{4}{|l|}{ OS } \\
\hline & & & $\mathrm{n}$ & $\mathrm{CR}+\mathrm{PR}$ & $\%$ & $\begin{array}{l}P \\
\text { value }\end{array}$ & OR $(95 \%$ CI $)$ & $\begin{array}{l}\text { Median } \\
\text { (month) }\end{array}$ & $\begin{array}{l}P \\
\text { value }\end{array}$ & HR $(95 \%$ CI $)$ & $\begin{array}{l}\text { Median } \\
\text { (month) }\end{array}$ & $\%$ & $\begin{array}{l}P \\
\text { value }\end{array}$ & HR $(95 \%$ CI) \\
\hline \multicolumn{15}{|l|}{ First-line Therapy } \\
\hline \multirow[t]{2}{*}{ Crinò et al, 2008} & G & 97 & 97 & 3 & 3.1 & NR & $0.60(0.14-2.58)$ & 2.7 & 0.310 & $\begin{array}{l}1.19 \\
(0.85-1.65)\end{array}$ & 5.9 & 33.9 & NR & $\begin{array}{l}0.98 \\
(0.66-1.47)\end{array}$ \\
\hline & $\mathrm{V}$ & 99 & 99 & 5 & 5.1 & & & 2.9 & & & 8.0 & 33.2 & & \\
\hline \multirow[t]{2}{*}{ Goss et al, 2009} & G & 100 & 100 & 6 & 6.0 & NR & $6.38(0.75-54.02)$ & NR & 0.217 & $\begin{array}{l}0.82 \\
(0.60-1.12)\end{array}$ & 3.7 & NR & 0.272 & $\begin{array}{l}0.84 \\
(0.62-1.15)\end{array}$ \\
\hline & $\mathrm{P}$ & 101 & 101 & 1 & 1.0 & & & NR & & & 2.8 & NR & & \\
\hline \multirow[t]{2}{*}{ Mok et al, 2009} & G & 609 & 609 & 262 & 43.0 & $<0.001$ & $1.59(1.26-2.01)$ & 5.7 & $<0.001$ & $\begin{array}{l}0.74 \\
(0.65-0.85)\end{array}$ & 18.6 & NR & NR & $\begin{array}{l}0.91 \\
(0.76-1.10)\end{array}$ \\
\hline & $\mathrm{PA}+\mathrm{CB}$ & 608 & 608 & 196 & 32.2 & & & 5.8 & & & 17.3 & NR & & \\
\hline \multirow[t]{2}{*}{$\begin{array}{l}\text { Maemondo et al, } \\
2010\end{array}$} & G & 114 & 114 & 84 & 73.7 & $<0.001$ & $6.32(3.55-11.25)$ & 10.8 & $<0.001$ & $\begin{array}{l}0.30 \\
(0.22-0.41)\end{array}$ & 30.5 & 61.4 & 0.310 & $\begin{array}{l}0.80 \\
(0.52-1.23)\end{array}$ \\
\hline & $\mathrm{PA}+\mathrm{CB}$ & 114 & 114 & 35 & 30.7 & & & 5.4 & & & 23.6 & 46.7 & & \\
\hline \multirow[t]{2}{*}{$\begin{array}{l}\text { Mitsudomi et al, } \\
2010\end{array}$} & G & 86 & 58 & 36 & 62.1 & $<0.001$ & 3.44 (1.61-7.38) & 9.2 & $<0.001$ & $\begin{array}{l}0.49 \\
(0.34-0.71)\end{array}$ & 30.9 & 80.2 & 0.211 & $\begin{array}{l}1.64 \\
(0.75-3.58)\end{array}$ \\
\hline & $\mathrm{D}+\mathrm{CS}$ & 86 & 59 & 19 & 32.2 & & & 6.3 & & & Not Reached & 88.4 & & \\
\hline \multirow[t]{2}{*}{ Han et al, 2012} & G & 159 & 159 & 88 & 55.4 & 0.101 & $1.45(0.93-2.28)$ & 5.8 & 0.138 & $\begin{array}{l}1.20 \\
(0.94-1.52)\end{array}$ & 22.3 & 47.7 & 0.604 & $\begin{array}{l}0.93 \\
(0.72-1.21)\end{array}$ \\
\hline & GP & 150 & 150 & 69 & 46.0 & & & 6.4 & & & 22.9 & 47.4 & & \\
\hline \multirow[t]{2}{*}{ Xie et al, 2015} & G & 27 & 27 & 15 & 55.6 & 0.711 & $0.80(0.26-2.49)$ & 8.0 & 0.293 & NR & NR & NR & NR & NR \\
\hline & $\mathrm{E}$ & 23 & 23 & 14 & 60.9 & & & 8.5 & & & NR & & & \\
\hline \multirow[t]{2}{*}{ Park et al, 2016} & G & 159 & 159 & 89 & 56.0 & 0.008 & $0.54(0.34-0.86)$ & 10.9 & 0.017 & $\begin{array}{l}1.37 \\
(1.05-1.75)\end{array}$ & 25.0 & NR & 0.330 & $\begin{array}{l}1.15 \\
(0.87-1.52)\end{array}$ \\
\hline & A & 160 & 160 & 112 & 70.0 & & & 11.0 & & & 27.9 & NR & & \\
\hline \multicolumn{15}{|c|}{ Non-first-line Therapy } \\
\hline \multirow[t]{2}{*}{ Cufer et al, 2006} & G & 68 & 68 & 9 & 13.2 & NR & $0.98(0.47-2.03)$ & 3.0 & 0.760 & $\begin{array}{l}0.94 \\
(0.64-1.39)\end{array}$ & 7.5 & 65.6 & 0.880 & $\begin{array}{l}0.97 \\
(0.61-1.52)\end{array}$ \\
\hline & $\mathrm{D}$ & 73 & 73 & 10 & 13.7 & & & 3.4 & & & 7.1 & 56.1 & & \\
\hline \multirow[t]{2}{*}{$\begin{array}{l}\text { Maruyama et al, } \\
2008\end{array}$} & G & 245 & 200 & 45 & 22.5 & 0.009 & $2.14(1.21-3.78)$ & 2.0 & 0.335 & $\begin{array}{l}0.90 \\
(0.72-1.12)\end{array}$ & 11.5 & 47.8 & 0.330 & $\begin{array}{l}1.12 \\
(0.89-1.40)\end{array}$ \\
\hline & $\mathrm{D}$ & 244 & 187 & 24 & 12.8 & & & 2.0 & & & 14.0 & 53.7 & & \\
\hline \multirow[t]{2}{*}{ Kim et al, 2008} & G & 733 & 659 & 60 & 9.1 & 0.330 & $1.22(0.82-1.84)$ & 2.2 & 0.470 & $\begin{array}{l}1.04 \\
(0.93-1.18)\end{array}$ & 7.6 & 32.0 & NR & $\begin{array}{l}1.02 \\
(0.91-1.15)\end{array}$ \\
\hline & $\mathrm{D}$ & 733 & 657 & 50 & 7.6 & & & 2.7 & & & 8.0 & 34.0 & & \\
\hline \multirow[t]{2}{*}{ Natale et al, 2009} & G & 85 & 85 & 1 & 1.2 & NR & NA & 1.9 & 0.025 & $\begin{array}{l}1.45 \\
(1.04-2.00)\end{array}$ & 7.4 & NR & 0.340 & $\begin{array}{l}0.84 \\
(0.60-1.19)\end{array}$ \\
\hline & V & 83 & 83 & 7 & 8.4 & & & 2.6 & & & 6.1 & NR & & \\
\hline \multirow[t]{2}{*}{ Lee et al, 2010} & G & 82 & 82 & 23 & 28.1 & 0.001 & NA & 3.3 & 0.044 & $\begin{array}{l}0.73 \\
(0.53-1.00)\end{array}$ & 14.1 & 18.3 & 0.437 & $\begin{array}{l}0.87 \\
(0.61-1.24)\end{array}$ \\
\hline & $\mathrm{D}$ & 79 & 79 & 6 & 7.6 & & & 3.4 & & & 12.2 & 25.3 & & \\
\hline Gaafar et al, 2011 & G & 86 & 86 & 10 & 12.0 & 0.0040 & NA & 4.1 & 0.002 & $\begin{array}{l}0.61 \\
(0.45-0.83)\end{array}$ & 10.9 & NR & 0.204 & $\begin{array}{l}0.81 \\
(0.59-1.12)\end{array}$ \\
\hline & $\mathrm{P}$ & 87 & 87 & 1 & 1.0 & & & 2.9 & & & 9.4 & NR & & \\
\hline Zhang et al, 2012 & G & 148 & 148 & 35 & 24.0 & 0.0001 & $54.10(7.17-408.00)$ & 4.8 & $<0.001$ & $\begin{array}{l}0.42 \\
(0.33-0.55)\end{array}$ & 18.7 & 46.6 & 0.260 & $\begin{array}{l}0.84 \\
(0.62-1.14)\end{array}$ \\
\hline & $\mathrm{P}$ & 148 & 148 & 1 & 1.0 & & & 2.6 & & & 16.9 & 37.2 & & \\
\hline Sun et al, 2012 & G & 68 & 68 & 40 & 58.8 & $<0.001$ & NA & 9.0 & 0.001 & $\begin{array}{l}0.54 \\
(0.37-0.79)\end{array}$ & 22.2 & NR & 0.370 & $\begin{array}{l}0.80 \\
(0.50-1.30)\end{array}$ \\
\hline & PE & 67 & 67 & 15 & 22.4 & & & 3.0 & & & 18.9 & NR & & \\
\hline Shi et al, 2013 & G & 196 & 196 & 53 & 27.2 & 0.910 & $0.98(0.63-1.52)$ & 3.4 & 0.130 & $\begin{array}{l}1.19 \\
(0.95-1.50)\end{array}$ & 13.9 & 19.4 & 0.570 & $\begin{array}{l}0.98 \\
(0.79-1.22)\end{array}$ \\
\hline & I & 199 & 199 & 55 & 27.6 & & & 4.6 & & & 13.3 & 16.6 & & \\
\hline Zhou et al, 2014 & G & 81 & 81 & 11 & 13.6 & 0.938 & NA & 1.6 & $<0.001$ & $\begin{array}{l}1.85 \\
(1.33-2.50)\end{array}$ & 9.6 & 76.5 & 0.077 & $\begin{array}{l}1.39 \\
(0.96-2.04)\end{array}$ \\
\hline & PE & 76 & 76 & 10 & 13.2 & & & 4.8 & & & 12.4 & 67.1 & & \\
\hline Yang et al, 2017 & G & 128 & 128 & 67 & 52.3 & 0.530 & NA & 10.4 & 0.108 & $\begin{array}{l}1.23 \\
(0.95-1.61)\end{array}$ & 20.1 & 28.1 & 0.250 & $\begin{array}{l}1.19 \\
(0.88-1.59)\end{array}$ \\
\hline & $\mathrm{E}$ & 128 & 128 & 76 & 56.3 & & & 13.0 & & & 22.9 & 28.1 & & \\
\hline
\end{tabular}

Abbreviations: NSCLC: non-small cell lung cancer; ITT: intention-to-treat; ORR: object response rate; CR: complete response; PR: partial response; PFS: progression-free survival; OR: odds ratio; OS: overall survival; HR: hazard ratio; G: gefitinib; V: vandetanib; PA: paclitaxel; CB: carboplatin; GP: gemcitabine + cisplatin ; E: erlotinib; A: afatinib; D: docetaxel; P: placebo; PE: pemetrexed; I: icotinib; CS: cisplatin; NR: not reported. 


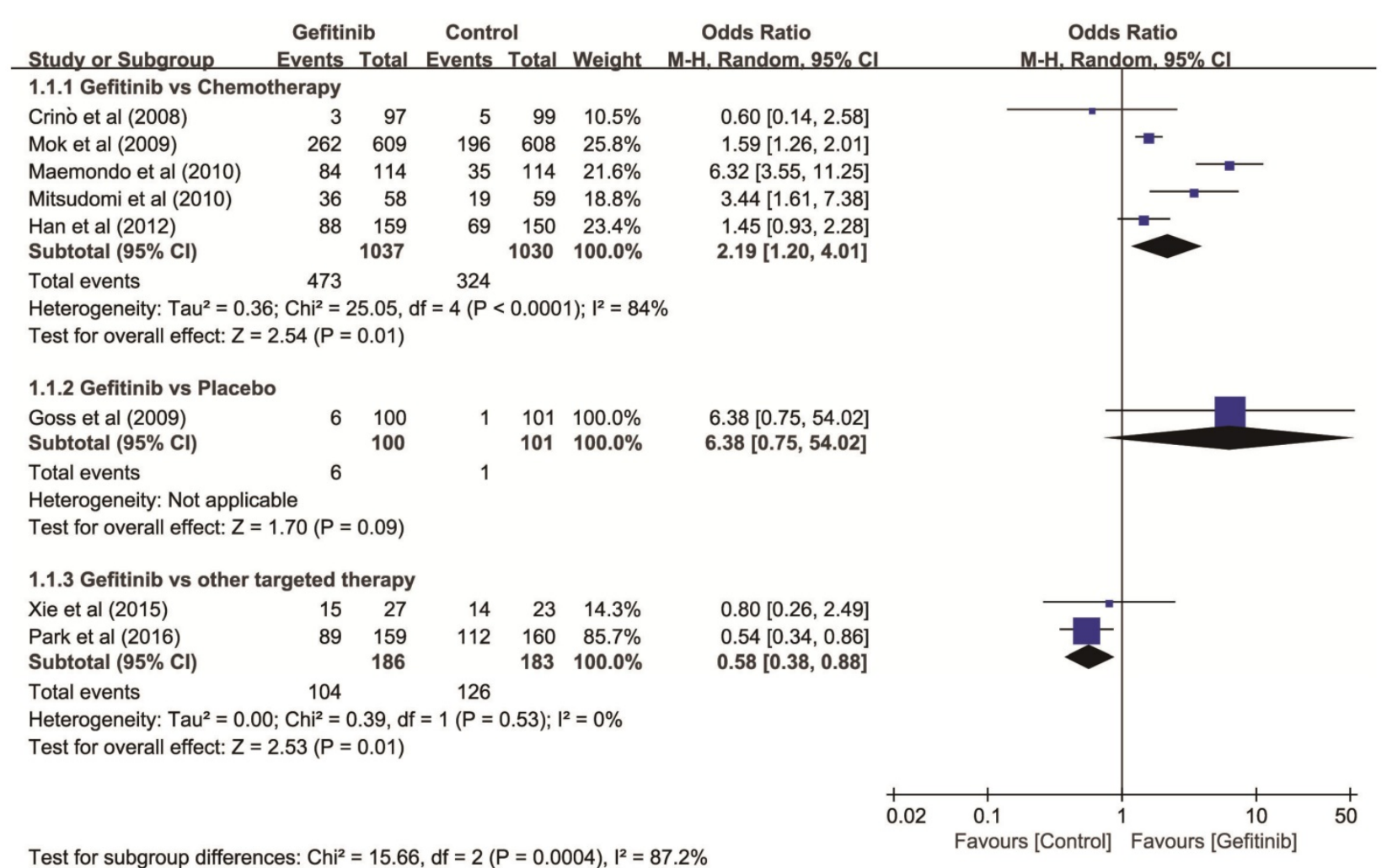

Figure 2. Forest plot of ORR of gefitinib compared to controls as first-line therapy for NSCLC. An OR of $>1$ indicates that the arm with the gefitinib performed better. Abbreviations: Cl: confidence interval; OR: odds ratio; ORR: objective response rate; NSCLC: non-small cell lung cancer.

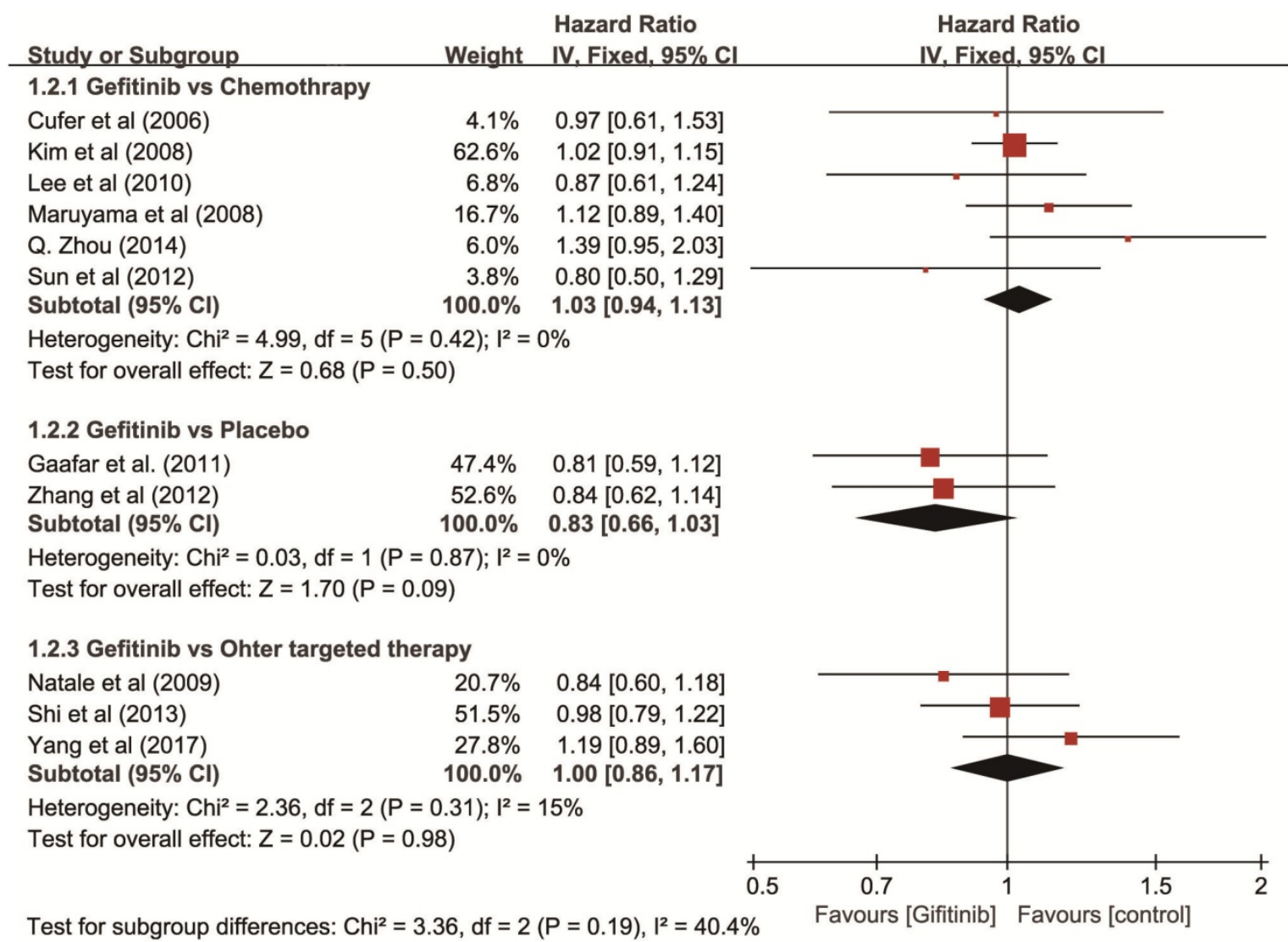

Figure 3. Forest plot of HR for OS of gefitinib compared to controls in non-first-line therapy for NSCLC. The heterogeneity test did not yield a significant result $(P=0.41)$. An HR of < 1 indicates that the arm with the gefitinib performed better. Abbreviations: Cl: confidence interval; HR: hazard ratio; OS: overall survival; NSCLC: non-small cell lung cancer. 


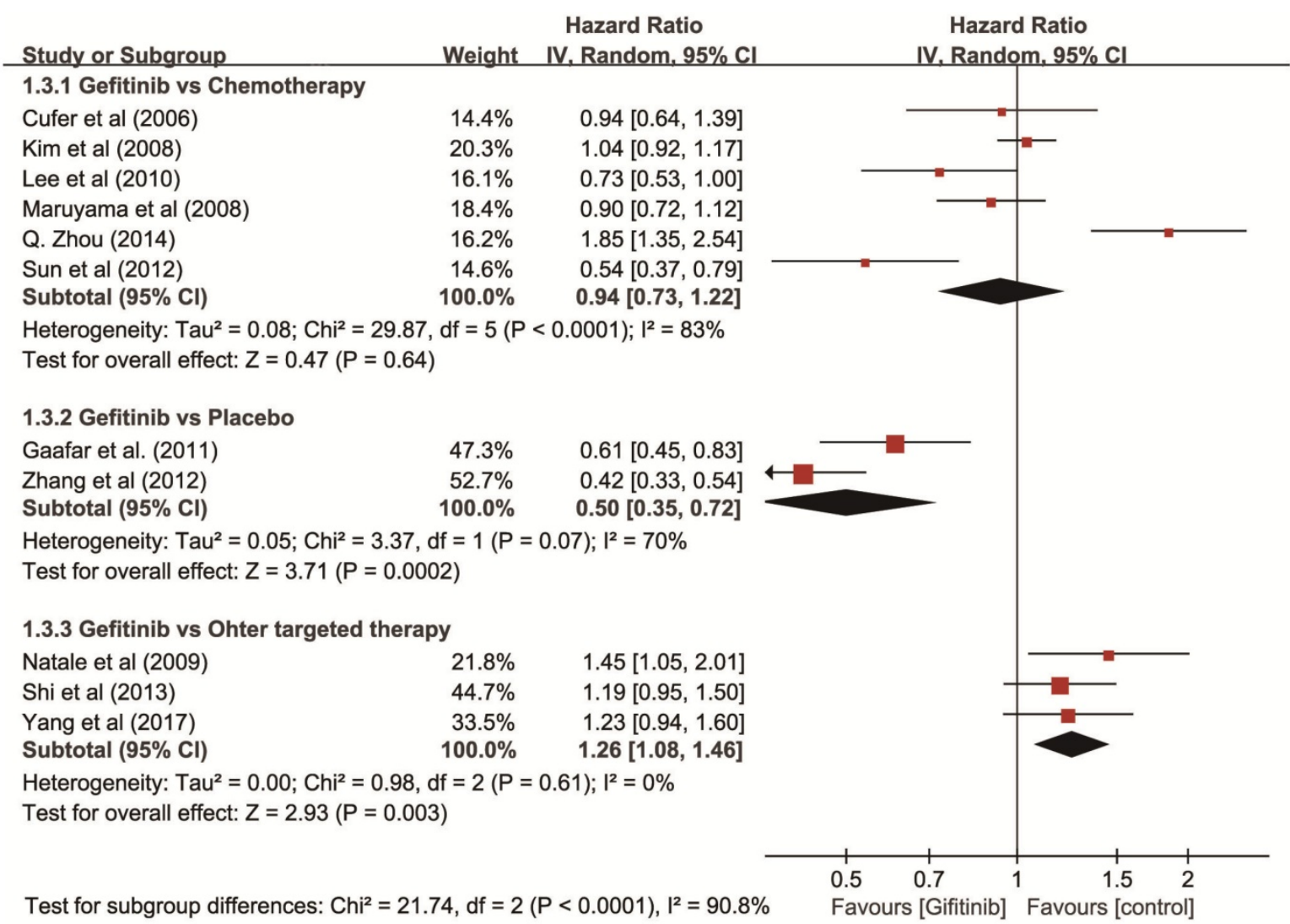

Figure 4. Forest plot of HR for PFS of gefitinib compared to controls in non-first-line therapy for NSCLC. An HR of $<1$ indicates that the arm with the gefitinib performed better. Abbreviations: Cl: confidence interval; HR: hazard ratio; PFS: progression-free survival; NSCLC: non-small cell lung cancer.

As first-line therapy, there was no statistically significant difference compared with gefitinib and placebo for ORR. It may be due to the sample size of the trail was limited. The patients in this study were in advanced stage or low strength grade, had short expected survival time so that the placebo was adopted as control. So gefitinib also cannot effectively inhibit tumor progression in this situation.

In non-first-line therapy, gefitinib effectively improved the PFS compared with placebo; but gefitinib appeared to be less efficient than other targeted therapies. Gefitinib showed similar PFS compared with chemotherapy. Comparing with placebo, chemotherapy and other targeted therapies, gefitinib showed similar in OS, respectively. Our results, together with other studies $[9,40,41]$, implied that there was no evidence that gefitinib improved overall survival compared with other therapies.

In toxicity analysis, patients who received gefitinib tended to reduce the incidence of hematologic toxicity, alopecia, neurotoxicity, etc. However, gefitinib appeared higher incidence of dry skin, abnormal hepatic function, rash, pruritus and diarrhea.

This study included nineteen articles, which vary from methodology quality and result completeness: (1) Randomization was incomplete.
Nine RCTs applied insufficient randomization. (2) Ten RCTs don't mention the allocation concealment, which may cause selective bias. One study showed the decrease in odds can be exaggerated 30\% when allocation concealment is unclear, and $41 \%$ when allocation concealment is insufficient [42]. (3) Only five RCTs adopted double-blind method. The use of blinding method makes sense for the objectivity and reliability of outcome measures. While owing to the control drugs of eleven RCTs were injections, they were difficult to compare with tablet gefitinib in blinding method.

Our meta-analysis has a higher power in illustrating the clinical implication of gefitinib compared to previously reported RCTs and meta-analyses, and will help make evidence-based treatment decisions, the design of future clinical trials, and understanding the variation of EGFR mutation for the treatment of NSCLC.

However, there were several limitations in this meta-analysis. First, the meta-analysis was limited to the data presented by authors of the source studies, in some cases the information of disease status, smoking status and pathological subtype was incomplete thus cannot be used for subgroup analysis. Secondly, our meta-analysis is heterogeneous regarding the research designs, diversity of patients and therapies, and this 
may lead to unreliable findings. The follow-up time of some studies is short, different median ages and races of patients, different drugs vary from dosage and duration of treatment, to some extent may cause clinical heterogeneity. To address this issue, we performed quality assessment and subgroup analysis. The quality assessment didn't invalidate the results of the meta-analysis. We also carried subgroup analysis in all efficient evaluation, and therefore we consider our results based on these trails believable. And then, since the numbers of studies include in each pooling are limited, the power of tests of publication bias is a concern. Therefore, publication bias could not be completely excluded. So we used trim and fill method to estimate the pooled association to address this concern. Finally, due to limited numbers of studies, the results of stratified analyses may have limited power to detect potential effect.

In conclusion, we updated the evidence of RCTs of gefitinib versus other agent-based regimens in treating NSCLC, considering the effect of treatment settings and EGFR mutation status. However, due to the limitations of included RCTs, more trials of higher quality, as well as longer-term follow-up are needed to confirm the efficacy and toxicity of gefitinib in NSCLC.

\section{Supplementary Material}

Supplementary figures and tables.

http://www.jcancer.org/v09p1455s1.pdf

\section{Acknowledgments}

This work was supported by two grants from the National Natural Science Foundation of China (NSFC81202283 to HGY, and 81773554 to HY) and the Priority Academic Program for the Development of Jiangsu Higher Education Institutions (Public Health and Preventive Medicine).

\section{Author Contributions}

YHG and CF conceived and designed the study. WHM and HJ drafted the manuscript; WHM and ZY searched databases and reviewed all articles. WHM and YH extracted the data. YHG and CF contributed reagents/materials/analysis tools. WHM carried out the statistical analysis and prepared the manuscript. All authors reviewed, critically revised, and approved the manuscript.

\section{Consent for publication} authors.

Publication consent was obtained from all

\section{Competing Interests}

The authors have declared that no competing interest exists.

\section{References}

1. Mao Y, Yang D, He J, Krasna MJ. Epidemiology of Lung Cancer. Surg Oncol Clin N Am. 2016; 25(3):439-445.

2. Siegel RL, Miller KD, Jemal A. Cancer statistics, 2016. CA Cancer J Clin. 2016; 66(1):7-30.

3. Sathiakumar N, Delzell E, Morrisey MA, Falkson C, Yong M, Chia V, et al. Mortality following bone metastasis and skeletal-related events among patients 65 years and above with lung cancer: A population-based analysis of U.S. Medicare beneficiaries, 1999-2006. Lung India. 2013; 30(1):20-26.

4. Cimino GD, Pan CX, Henderson PT. Personalized medicine for targeted and platinum-based chemotherapy of lung and bladder cancer. Bioanalysis. 2013; 5(3):369-391.

5. Crino L, Metro G. Therapeutic options targeting angiogenesis in nonsmall cell lung cancer. Eur Respir Rev. 2014; 23(131):79-91.

6. Liu TC, Jin X, Wang Y, Wang K. Role of epidermal growth factor receptor in lung cancer and targeted therapies. Am J Cancer Res. 2017; 7(2):187-202.

7. Ettinger DS, Wood DE, Akerley W, Bazhenova LA, Borghaei H, Camidge DR, et al. NCCN Guidelines Insights: Non-Small Cell Lung Cancer, Version 4.2016. J Natl Compr Canc Netw. 2016; 14(3):255-264.

8. Tan PS, Bilger M, de Lima LG, Acharyya S, Haaland B. Meta-analysis of first-line therapies with maintenance regimens for advanced non-small-cell lung cancer (NSCLC) in molecularly and clinically selected populations. Cancer Med. 2017; 6(8):1847-1860

9. Haaland B, Tan PS, de Castro GJ, Lopes G. Meta-analysis of first-line therapies in advanced non-small-cell lung cancer harboring EGFR-activating mutations. J Thorac Oncol. 2014; 9(6):805-811.

10. Ellis PM, Coakley N, Feld R, Kuruvilla S, Ung YC. Use of the epidermal growth factor receptor inhibitors gefitinib, erlotinib, afatinib, dacomitinib, and icotinib in the treatment of non-small-cell lung cancer: a systematic review. Curr Oncol. 2015; 22(3):e183-e215.

11. Hong D, Zhang G, Zhang X, Lian X. Pulmonary Toxicities of Gefitinib in Patients With Advanced Non-Small-Cell Lung Cancer: A Meta-Analysis of Randomized Controlled Trials. Medicine (Baltimore). 2016; 95(9):e3008.

12. Higgins JPT. Cochrane Handbook for Systematic Reviews of Interventions : A Handbook: John Wiley \& Sons, Incorporated; 2010.

13. Tierney JF, Stewart LA, Ghersi D, Burdett S, Sydes MR. Practical methods for incorporating summary time-to-event data into meta-analysis. Trials. 2007; 8:16.

14. DerSimonian R, Laird N. Meta-analysis in clinical trials revisited. Contemp Clin Trials. 2015; 45(Pt A):139-145.

15. Begg CB, Mazumdar M. Operating characteristics of a rank correlation test for publication bias. Biometrics. 1994; 50(4):1088-1101.

16. Egger M, Davey SG, Schneider M, Minder C. Bias in meta-analysis detected by a simple, graphical test. BMJ. 1997; 315(7109):629-634

17. Duval S, Tweedie R. Trim and fill: A simple funnel-plot-based method of testing and adjusting for publication bias in meta-analysis. Biometrics. 2000; 56(2):455-463.

18. Knobloch K, Yoon U, Vogt PM. Preferred reporting items for systematic reviews and meta-analyses (PRISMA) statement and publication bias. J Craniomaxillofac Surg. 2011; 39(2):91-92

19. Crino L, Cappuzzo F, Zatloukal P, Reck M, Pesek M, Thompson JC, et al. Gefitinib versus vinorelbine in chemotherapy-naive elderly patients with advanced non-small-cell lung cancer (INVITE): a randomized, phase II study. J Clin Oncol. 2008; 26(26):4253-4260.

20. Goss G, Ferry D, Wierzbicki R, Laurie SA, Thompson J, Biesma B, et al. Randomized phase II study of gefitinib compared with placebo in chemotherapy-naive patients with advanced non-small-cell lung cancer and poor performance status. J Clin Oncol. 2009; 27(13):2253-2260.

21. Mok TS, Wu YL, Thongprasert S, Yang CH, Chu DT, Saijo N, et al. Gefitinib or carboplatin-paclitaxel in pulmonary adenocarcinoma. N Engl J Med. 2009; 361(10):947-957.

22. Maemondo $M$, Inoue $A$, Kobayashi $K$, Sugawara $S$, Oizumi $S$, Isobe $H$, et al. Gefitinib or chemotherapy for non-small-cell lung cancer with mutated EGFR. N Engl J Med. 2010; 362(25):2380-2388.

23. Mitsudomi T, Morita S, Yatabe Y, Negoro S, Okamoto I, Tsurutani J, et al. Gefitinib versus cisplatin plus docetaxel in patients with non-small-cell lung cancer harbouring mutations of the epidermal growth factor receptor (WJTOG3405): an open label, randomised phase 3 trial. Lancet Oncol. 2010; 11(2):121-128.

24. Han JY, Park K, Kim SW, Lee DH, Kim HY, Kim HT, et al. First-SIGNAL: first-line single-agent iressa versus gemcitabine and cisplatin trial in never-smokers with adenocarcinoma of the lung. J Clin Oncol. 2012; 30(10):1122-1128.

25. Xie Y, Liang J, Su N. [Gefitinib versus Erlotinib as first-line treatment for patients with advanced EGFR mutation-positive non-small-cell lung cancer]. Nan Fang Yi Ke Da Xue Xue Bao. 2015; 35(3):446-449.

26. Park K, Tan EH, O'Byrne K, Zhang L, Boyer M, Mok T, et al. Afatinib versus gefitinib as first-line treatment of patients with EGFR mutation-positive non-small-cell lung cancer (LUX-Lung 7): a phase 2B, open-label, randomised controlled trial. Lancet Oncol. 2016; 17(5):577-589. 
27. Cufer T, Vrdoljak E, Gaafar R, Erensoy I, Pemberton K. Phase II, open-label, randomized study (SIGN) of single-agent gefitinib (IRESSA) or docetaxel as second-line therapy in patients with advanced (stage IIIb or IV) non-small-cell lung cancer. Anticancer Drugs. 2006; 17(4):401-409.

28. Maruyama R, Nishiwaki $Y$, Tamura T, Yamamoto N, Tsuboi M, Nakagawa K, et al. Phase III study, V-15-32, of gefitinib versus docetaxel in previously treated Japanese patients with non-small-cell lung cancer. J Clin Oncol. 2008; 26(26):4244-4252.

29. Kim ES, Hirsh V, Mok T, Socinski MA, Gervais R, Wu YL, et al. Gefitinib versus docetaxel in previously treated non-small-cell lung cancer (INTEREST): a randomised phase III trial. Lancet. 2008; 372(9652):1809-1818.

30. Natale RB, Bodkin D, Govindan R, Sleckman BG, Rizvi NA, Capo A, et al. Vandetanib versus gefitinib in patients with advanced non-small-cell lung cancer: results from a two-part, double-blind, randomized phase ii study. J Clin Oncol. 2009; 27(15):2523-2529.

31. Lee DH, Park K, Kim JH, Lee JS, Shin SW, Kang JH, et al. Randomized Phase III trial of gefitinib versus docetaxel in non-small cell lung cancer patients who have previously received platinum-based chemotherapy. Clin Cancer Res. 2010; 16(4):1307-1314.

32. Gaafar RM, Surmont VF, Scagliotti GV, Van Klaveren RJ, Papamichael D, Welch JJ, et al. A double-blind, randomised, placebo-controlled phase III intergroup study of gefitinib in patients with advanced NSCLC, non-progressing after first line platinum-based chemotherapy (EORTC 08021/ILCP 01/03). Eur J Cancer. 2011; 47(15):2331-2340.

33. Zhang L, Ma S, Song X, Han B, Cheng Y, Huang C, et al. Gefitinib versus placebo as maintenance therapy in patients with locally advanced or metastatic non-small-cell lung cancer (INFORM; C-TONG 0804): a multicentre, double-blind randomised phase 3 trial. Lancet Oncol. 2012; 13(5):466-475.

34. Sun JM, Lee KH, Kim SW, Lee DH, Min YJ, Yun HJ, et al. Gefitinib versus pemetrexed as second-line treatment in patients with nonsmall cell lung cancer previously treated with platinum-based chemotherapy (KCSG-LU08-01): an open-label, phase 3 trial. Cancer-Am Cancer Soc. 2012; 118(24):6234-6242.

35. Shi $Y$, Zhang L, Liu X, Zhou C, Zhang L, Zhang S, et al. Icotinib versus gefitinib in previously treated advanced non-small-cell lung cancer (ICOGEN): a randomised, double-blind phase 3 non-inferiority trial. Lancet Oncol. 2013; 14(10):953-961.

36. Zhou Q, Cheng Y, Yang JJ, Zhao MF, Zhang L, Zhang XC, et al. Pemetrexed versus gefitinib as a second-line treatment in advanced nonsquamous nonsmall-cell lung cancer patients harboring wild-type EGFR (CTONG0806): a multicenter randomized trial. Ann Oncol. 2014; 25(12):2385-2391.

37. Yang JJ, Zhou Q, Yan HH, Zhang XC, Chen HJ, Tu HY, et al. A phase III randomised controlled trial of erlotinib vs gefitinib in advanced non-small cell lung cancer with EGFR mutations. Br J Cancer. 2017; 116(5):568-574.

38. Tetsu O, Hangauer MJ, Phuchareon J, Eisele DW, McCormick F. Drug Resistance to EGFR Inhibitors in Lung Cancer. Chemotherapy. 2016; 61(5):223-235.

39. Kuykendall A, Chiappori A. Advanced EGFR mutation-positive non-small-cell lung cancer: case report, literature review, and treatment recommendations. Cancer Control. 2014; 21(1):67-73.

40. Inoue A, Kobayashi K, Maemondo M, Sugawara S, Oizumi S, Isobe H, et al. Updated overall survival results from a randomized phase III trial comparing gefitinib with carboplatin-paclitaxel for chemo-naive non-small cell lung cancer with sensitive EGFR gene mutations (NEJ002). Ann Oncol. 2013; 24(1):54-59.

41. Paz-Ares L, Tan EH, O'Byrne K, Zhang L, Hirsh V, Boyer M, et al. Afatinib versus gefitinib in patients with EGFR mutation-positive advanced non-small-cell lung cancer: overall survival data from the phase IIb LUX-Lung 7 trial. Ann Oncol. 2017; 28(2):270-277.

42. Hirst JA, Howick J, Aronson JK, Roberts N, Perera R, Koshiaris C, et al. The need for randomization in animal trials: an overview of systematic reviews. Plos One. 2014; 9(6):e98856. 